JOURNAL OF THE

AMERICAN MATHEMATICAL SOCIETY

Volume 16, Number 2, Pages 443-459

S 0894-0347(02)00415-0

Article electronically published on December 11, 2002

\title{
CLASSIFICATION OF LIMITING SHAPES FOR ISOTROPIC CURVE FLOWS
}

\author{
BEN ANDREWS
}

\section{INTRODUCTION}

Some natural parabolic deformations of convex curves in the plane are those in which each point moves in a direction normal to the curve with speed equal to a power of the curvature: That is, given a convex curve $\gamma$ which is the image of an embedding $x_{0}: S^{1} \rightarrow \mathbb{R}^{2}$, the deformation is obtained by solving the equation

$$
\frac{\partial x}{\partial t}=-\frac{1}{\alpha} \kappa^{\alpha} \mathbf{n}
$$

with $\alpha \neq 0$, and initial condition $x(p, 0)=x_{0}(p)$. This produces a family of curves $\gamma_{t}=x\left(S^{1}, t\right)$. Here $\kappa$ is the curvature, and $\mathbf{n}$ is the outward-pointing unit normal vector. These equations are particularly natural in that they are isotropic (equivariant under rotations in the plane) and homogeneous (equivariant under dilation of space, if time is also scaled accordingly).

The main aim of this paper is to provide a complete description of the behaviour of embedded convex curves moving by equations of the form (1.1). In certain cases this description has already been provided: If $\alpha=1$ then Equation (1.1) is the curve-shortening flow, for which the following holds:

Theorem 1.1. Let $\alpha=1$ and let $\gamma_{0}$ be a smooth convex embedded closed curve given by an embedding $x_{0}: S^{1} \rightarrow \mathbb{R}^{2}$. Then there exists a unique solution $x: S^{1} \times$ $[0, T) \rightarrow \mathbb{R}^{2}$ of the curve-shortening flow (1.1) with initial data $x_{0}$, and $\gamma_{t}=x\left(S^{1}, t\right)$ converges to a point $p \in \mathbb{R}^{2}$ as $t \rightarrow T$. The rescaled curves $\left(\gamma_{t}-p\right) / \sqrt{2(T-t)}$ converge to the unit circle about the origin as $t \rightarrow T$.

This result was first proved by Gage and Hamilton (11], 12], [15]). The assumption of smooth initial data can be relaxed to allow any curve $\gamma_{0}$ which is the boundary of a bounded open convex region, in which case the curves $\gamma_{t}$ approach $\gamma_{0}$ in Hausdorff distance as $t \rightarrow 0$ [2, Theorem II2.8]. Furthermore, Grayson [14] proved that the assumption of convexity of the initial curve can be removed, and the result holds for arbitrary smooth embedded initial curves. More recent proofs of Grayson's theorem have been given by Hamilton [16] and by Huisken [17]. Similar results for anisotropic analogues of this flow appear in Oaks [18] and Chou and Zhu [10].

Another case which is well understood is that with $\alpha=1 / 3$. In this case the results reflect a surprising affine invariance property of the equation:

Received by the editors November 4, 2002.

2000 Mathematics Subject Classification. Primary 53C44; Secondary 35K55, 53A04.

Research supported by a grant from the Australian Research Council.

(C)2002 American Mathematical Society 
Theorem 1.2. Let $\alpha=\frac{1}{3}$. If $\gamma_{0}=x_{0}\left(S^{1}\right)$ is a smooth convex closed curve given by an embedding $x_{0}: S^{1} \rightarrow \mathbb{R}^{2}$, then there exists a unique solution $x: S^{1} \times[0, T) \rightarrow \mathbb{R}^{2}$ of (1.1) with initial data $x_{0}$, and $\gamma_{t}=x\left(S^{1}, t\right)$ converges to a point $p \in \mathbb{R}^{2}$ as $t \rightarrow T$ while $\left(\gamma_{t}-p\right) /(4(T-t) / 3)^{3 / 4}$ converges to an ellipse of enclosed area $\pi$ centred at the origin.

The regularity assumptions on the initial curve can be relaxed to allow boundaries of open bounded convex regions. This result was first proved in [19] and [2] (see also [5, §9]). Results for non-convex curves appear in [7.

If $\alpha>1$, then the results are similar to those in Theorem 1.1, except for some differences in the regularity of solutions (see [4, Corollary II3.2]):

Theorem 1.3. Let $\alpha>1$ and let $\gamma_{0}$ be the boundary of an open bounded convex set in $\mathbb{R}^{2}$. Then there exists a solution $x: S^{1} \times[0, T) \rightarrow \mathbb{R}^{2}$ of Equation (1.1) with $x\left(S^{1}, t\right)$ converging to $\gamma_{0}$ in Hausdorff distance as $t \rightarrow 0$. This solution is unique up to time-independent reparametrisation, $C^{2+1 /(\alpha-1)}$ for positive times, and $C^{\infty}$ for times close to $T$. The curves $\gamma_{t}=x\left(S^{1}, t\right)$ converge to a point $p \in \mathbb{R}^{2}$ as $t \rightarrow T$ while $\left(\gamma_{t}-p\right) /((1+\alpha)(T-t))^{1 /(1+\alpha)}$ converges smoothly to the unit circle about the origin. If $\gamma_{0}$ is smooth and strictly convex, then the solution is smooth and strictly convex for all $t$.

For $\frac{1}{3}<\alpha<1$, complete results have not yet been given - the result available for more general anisotropic flows implies the following:

Theorem 1.4. Suppose $\alpha \in\left(\frac{1}{3}, 1\right]$ and $\gamma_{0}$ is the boundary curve of an open bounded convex set in $\mathbb{R}^{2}$. Then there exists a solution $x: S^{1} \times(0, T) \rightarrow \mathbb{R}^{2}$ of Equation (1.1) such that $\gamma_{t}=x\left(S^{1}, t\right)$ converge to $\gamma_{0}$ in Hausdorff distance as $t \rightarrow 0$. This solution is unique up to time-independent reparametrisation, and smooth and strictly convex for $t>0$. The curves $\gamma_{t}$ converge to $p \in \mathbb{R}^{2}$ as $t \rightarrow T$, and the rescaled curves $\left(\gamma_{t}-p\right) /(T-t)^{1 /(1+\alpha)}$ converge smoothly to a limit curve $\gamma_{T}$ which satisfies $\kappa^{\alpha}=$ $\lambda\langle x, \mathbf{n}\rangle$ for some $\lambda>0$.

This is proved in 4, Theorem II2.8], with the smooth convergence statement provided by [3, Theorem 2]. Curves which satisfy $\kappa^{\alpha}=\lambda\langle x, \mathbf{n}\rangle$ are called homothetic solutions for Equation (1.1), because the solution of (1.1) with this initial data is $\gamma_{t}=(1-(1+\alpha) \lambda t)^{1 /(1+\alpha)} \gamma_{0}$ (up to reparametrisation). Theorem 1.4 therefore reduces the study of the asymptotic behaviour of these flows to a classification of homothetic solutions. Some results for non-convex solutions are given in [9] Chapter 8].

The first main result of this paper is a classification of the homothetically shrinking embedded solutions of the flows (1.1):

Theorem 1.5. If $\alpha>\frac{1}{3}$ then the only embedded homothetically contracting solutions of (1.1) are circles with centre at the origin. If $\alpha=\frac{1}{3}$ then every ellipse centred at the origin is a homothetically contracting solution, and there are no others. If $\alpha \in\left[\frac{1}{8}, \frac{1}{3}\right)$ then the only embedded homothetically contracting solutions are circles centred at the origin. If $0<\alpha<\frac{1}{8}$ then the embedded homothetically contracting solutions are circles centred at the origin, as well as unique (up to rotation and scaling) curves $\Gamma_{k, \alpha}$ with $k$-fold symmetry, for each integer $k \geq 3$ with $k<\sqrt{\frac{\alpha+1}{\alpha}}$. The curves $\Gamma_{k, \alpha}$ depend smoothly on $\alpha<\frac{1}{k^{2}-1}$ and converge to regular $k$-sided polygons as $\alpha \searrow 0$ and to circles as $\alpha \nearrow \frac{1}{k^{2}-1}$. 
The first statement is also proved in 9 . With Theorem 1.4 we deduce:

Corollary 1.6. If $1 / 3<\alpha<1$ and $\gamma_{0}$ is any embedded convex closed curve, there exists a unique smooth solution of (1.1) with initial condition $\gamma_{0}$, and this contracts to a point in finite time while becoming circular in shape.

The remaining case is $\alpha \in\left(0, \frac{1}{3}\right)$. It is shown in [6] that the isoperimetric ratio becomes unbounded near the final time for generic symmetric initial data. Some implications of Theorem 1.5 for $\alpha<\frac{1}{3}$ are given in Section 7

In the case $\alpha<0$, a complete description of the behaviour of embedded convex solutions of (1.1) is known: This has been provided by Gerhardt 13 for $\alpha=-1$ and by Urbas [21], [22] for $-1 \leq \alpha<0$, and by Chow and Tsai [8], Tsai [20] and the author [4 Theorem I2.1] for $\alpha<-1$. In these cases all convex solutions expand to infinite size while becoming circular in shape. Similar results hold for more general nonhomogeneous expansion flows of curves, including some cases allowing non-convexity.

The methods of this paper also allow a classification of homothetic solutions without the assumption of embeddedness. Homothetic solutions which are $C^{\infty}$ immersed closed curves are always strictly locally convex, and are periodic, with the arcs between any two consecutive vertices being congruent. The result shows that homothetic solutions are determined by the total curvature between consecutive vertices, which must lie in a certain range:

Theorem 1.7. Let $m$ and $n$ be mutually prime. There exists a smooth, strictly locally convex, non-circular immersed homothetic solution $\Gamma_{n, m, \alpha}$ with total curvature $2 \pi n$ and $m$ maxima of curvature if and only if

$$
\begin{aligned}
& \sqrt{\frac{\alpha}{1+\alpha}}<\frac{n}{m}<\frac{1}{2}, \quad 0<\alpha<\frac{1}{3} ; \\
& \frac{1}{2}<\frac{n}{m}<\sqrt{\frac{\alpha}{1+\alpha}}, \quad \frac{1}{3}<\alpha \leq 1 \text {; } \\
& \frac{\alpha}{1+\alpha}<\frac{n}{m}<\sqrt{\frac{\alpha}{1+\alpha}}, \quad \alpha>1 \text {; } \\
& \sqrt{\frac{|\alpha|}{|\alpha|-1}}<\frac{n}{m}<\frac{|\alpha|}{|\alpha|-1}, \quad \alpha<-1 .
\end{aligned}
$$

In each of the above cases there is exactly one such curve up to rotation and scaling. If $\alpha=\frac{1}{3}$ then all homothetically contracting solutions are ellipses. If $\alpha \in[-1,0)$ there are no compact homothetic solutions other than circles.

The case $\alpha=1$ of the above theorem was previously proved in $[1]$ by a computerassisted argument, considerably more complicated than that given here. The case $\alpha=\frac{1}{3}$ was proved in [2, Lemma 6.1]. The existence parts of the theorem, but not the uniqueness or the nonexistence for other $m$ and $n$, are also proved in 23 . Theorem 1 and §4]. The statement in the case $-1 \leq \alpha<0$ is implicit in the argument of [21].

If $\alpha>1$ or $\alpha<-1$ then there are also compact homothetic solutions which are not $C^{\infty}$ and are not strictly locally convex. These have been discussed in [23], and will not appear again in this paper.

\section{THE PERIOD FUNCTIONS}

In this section the main theorems are reduced to estimates on certain functions defined by integrals. 
A homothetically shrinking solution of (1.1) with $\alpha>0$, or a homothetically expanding one with $\alpha<0$, satisfies the equation

$$
\kappa^{\alpha}=\lambda\langle x, \mathbf{n}\rangle
$$

for some $\lambda>0$. If $\alpha \neq-1$ then one can choose $\lambda=1$ by scaling the curve. On any portion of the curve where the curvature does not change sign, the Gauss map is a local diffeomorphism, so one can parametrise the curve to get $\mathbf{n}(z)=z$ everywhere, $z \in S^{1}$. Let $u$ be the support function, defined by $u(z)=\langle x(z), z\rangle$. Then the curvature is given by

$$
\kappa^{-1}=\frac{\partial^{2} u}{\partial \theta^{2}}+u,
$$

where $\theta$ is the arc-length parameter on $S^{1}$. The identity (2.1) becomes

$$
\frac{\partial^{2} u}{\partial \theta^{2}}+u=u^{-1 / \alpha} .
$$

This is a second-order ordinary differential equation for $u$, which can be solved to determine the shapes of all possible homothetic solutions. A first integral of (2.3) is given by

$$
\begin{aligned}
u_{\theta}^{2}+u^{2}-\frac{2 \alpha}{\alpha-1} u^{1-1 / \alpha} & =E, & & \alpha \neq 1 \\
u_{\theta}^{2}+u^{2}-\log u & =E, & & \alpha=1
\end{aligned}
$$

for some constant $E$. Note that if $u$ reaches zero, then the curvature of the curve reaches zero (if $\alpha>0$ ) or infinity (if $\alpha<0$ ). Otherwise the solution of Equation (2.3) is a periodic positive function of $\theta$, with maximum and minimum values of $u$ determined by $E$ and $\alpha$. In the latter case it is convenient to parametrise the solutions not by $E$ but by the ratio $r$ of the maximum and minimum values $u_{ \pm}$of $u$ : Equation (2.4) gives:

$$
\begin{array}{lll}
0=E-u_{ \pm}^{2}+\frac{2 \alpha}{\alpha-1} u_{ \pm}^{1+1 / \alpha}, & & \alpha \neq 1 \\
0=E-u_{ \pm}^{2}+2 \log u_{ \pm}, & & \alpha=1
\end{array}
$$

and therefore

$$
\begin{array}{ll}
E=\left(\frac{2 \alpha\left(r^{\frac{\alpha-1}{\alpha}}-1\right)}{(\alpha-1)\left(r^{2}-1\right)}\right)^{\frac{2 \alpha}{\alpha+1}}\left(\frac{r^{\frac{\alpha-1}{\alpha}}-r^{2}}{r^{\frac{\alpha-1}{\alpha}}-1}\right), & \alpha \neq 1 ; \\
E=\frac{2 \log r}{1-r^{-2}}-\frac{1}{2} \log \left(\frac{2 \log r}{1-r^{-2}}\right), & \alpha=1 .
\end{array}
$$

This is an increasing function of $r$ for $r \geq 1$, with $E \rightarrow \frac{1+\alpha}{1-\alpha}$ as $r \rightarrow 1$ for $\alpha \neq 1$ and $E \rightarrow 1$ as $r \rightarrow 1$ for $\alpha=1$, while $E \rightarrow \infty$ as $r \rightarrow \infty$ for $0<\alpha \leq 1$ and $E \rightarrow 0$ as $r \rightarrow \infty$ for $\alpha>1$ or $\alpha<-1$. This range therefore covers all of the solutions of Equation (2.4) if $0<\alpha \leq 1$, but excludes those with $E>0$ if $\alpha>1$. The latter have zero minimum value of $u$, at which $\left|u_{\theta}\right| \geq 0$, so it is natural to reparametrise by setting $v$ to be the ratio of the value of $\left|u_{\theta}\right|$ when $u=0$ to the maximum value of $u$.

Definition 2.1. For $r \in(1, \infty)$ and $\alpha \in(0, \infty) \backslash\{1\} \cup(-\infty,-1)$, define

$$
\Theta(\alpha, r)=\int_{1}^{r} \frac{d x}{\sqrt{\frac{r^{2}-r \frac{\alpha-1}{\alpha}}{1-r^{\frac{\alpha-1}{\alpha}}}-x^{2}-\frac{r^{2}-1}{1-r^{\frac{\alpha-1}{\alpha}}} x^{\frac{\alpha-1}{\alpha}}}} .
$$


If $\alpha=1$ then define for $r \in(1, \infty)$

$$
\Theta(1, r)=\int_{1}^{r} \frac{d x}{\sqrt{1-x^{2}+\frac{r^{2}-1}{\log r} \log x}} .
$$

If $\alpha \in(1, \infty) \cup(-\infty,-1)$ and $v \in(0, \infty) \backslash\{1\}$, define

$$
\Xi(\alpha, v)=\int_{0}^{1} \frac{d x}{\sqrt{\left(1-v^{2}\right) x^{\frac{\alpha-1}{\alpha}}+v^{2}-x^{2}}} .
$$

If $\alpha=1$ and $\lambda>0$, define

$$
\Psi(1, \lambda)=\int_{0}^{1} \frac{d x}{\sqrt{1-x^{2}-2 \lambda \log x}},
$$

and if $0<\alpha<1$ and $\lambda>0$, define

$$
\Psi(\alpha, \lambda)=\int_{0}^{1} \frac{d x}{\sqrt{1-x^{2}+\frac{2 \alpha \lambda}{1-\alpha}\left(u^{-\frac{1-\alpha}{\alpha}}-1\right)}} .
$$

$\Theta(\alpha, r)$ gives the total curvature of the unique (up to rotation) smooth, strictly locally convex homothetic curve segment with monotone curvature and endpoints tangent to the circles of radius 1 and $r$ about the origin.

If $0<v<1, \Xi(\alpha, v)$ gives the total curvature of the unique (up to rotation) homothetic locally convex curve segment with monotone curvature with one end tangent to the circle of radius one, and the other pointing towards the origin at a distance $v$. If $\alpha>1$ then the curvature approaches zero at the latter endpoint, while if $\alpha<-1$ then the curvature instead approaches infinity.

If $v>1$ then $\Xi(\alpha, v)$ gives the total curvature of the homothetically expanding (for $\alpha>1$ ) or contracting (for $\alpha<-1$ ) curve with monotone curvature, with one end tangent to the unit circle and the other pointing radially outward from the origin at distance $v$. The curvature at the latter end approaches zero for $\alpha>1$ and infinity for $\alpha<-1$.

$\Psi(\alpha, \lambda)$ gives the total curvature of the homothetically expanding curve with monotone curvature with one end tangent to the unit circle and the other approaching infinity asymptotic to a radial line from the origin. $\lambda$ gives the radius of curvature at the former endpoint.

The results stated in the introduction all follow from information about the values of $\Theta$ as $r \rightarrow 1$ and $r \rightarrow \infty$, together with statements on monotonicity of $\Theta$ in $\alpha$ and $r$. The next section will investigate the values of the period functions at all of the extreme arguments, and the following two sections will prove the required results on monotonicity.

\section{Duality and special values}

In this section a surprising symmetry is established in the values of the function $\Theta$, and values are computed for special choices of the arguments. The following proposition summarises the results: 
Theorem 3.1. $\Theta$ is continuous on $(0, \infty) \times(1, \infty)$ and on $(-\infty,-1) \times(1, \infty)$, and has the following limiting values:

$$
\begin{array}{lll}
\lim _{r \rightarrow 1} \Theta(\alpha, r)=\pi \sqrt{\frac{\alpha}{1+\alpha}}, & & \alpha \in(-\infty,-1) \cup(0, \infty) ; \\
\lim _{r \rightarrow \infty} \Theta(\alpha, r)=\frac{\pi}{2}, & & \alpha \in(0,1] ; \\
\lim _{r \rightarrow \infty} \Theta(\alpha, r)=\pi \frac{\alpha}{1+\alpha}, & & \alpha \in(-\infty,-1) \cup(1, \infty) ; \\
\lim _{\alpha \searrow 0} \Theta(\alpha, r)=\arccos (1 / r) ; & & \\
\lim _{\alpha \rightarrow \infty} \Theta(\alpha, r)=\pi ; & & \\
\lim _{\alpha \rightarrow-\infty} \Theta(\alpha, r)=\pi ; & & \\
\lim _{\alpha \nearrow-1} \Theta(\alpha, r) & =\infty . &
\end{array}
$$

Furthermore the following symmetry holds:

$$
\Theta\left(\frac{\alpha+1}{3 \alpha-1}, r^{\frac{1+\alpha}{2 \alpha}}\right)=\frac{1+\alpha}{2 \alpha} \Theta(\alpha, r)
$$

for any $\alpha \in \mathbb{R}$ with $\alpha \neq 1 / 3$ and $\alpha \notin[-1,0]$ and $r>1$.

Proof. The continuity is standard, and the limiting values can be deduced without difficulty. The symmetry (3.1) is proved as follows: Assume $\alpha \neq 1$, since the symmetry is trivial for $\alpha=1$. Let $\Theta=\Theta(\alpha, r)$, and suppose $u:[0, \Theta] \rightarrow \mathbb{R}$ is the monotone solution of (2.3) with $u^{\prime}(0)=u^{\prime}(\Theta)=0$ and $u(\Theta)=r u(0)$. Let $w:\left[0, \frac{1+\alpha}{2 \alpha} \Theta\right] \rightarrow \mathbb{R}$ be defined by $w(\theta)=u\left(\frac{2 \alpha}{1+\alpha} \theta\right)^{\frac{1+\alpha}{2 \alpha}}$. Then $w$ satisfies the identity

$$
\begin{aligned}
\left(\frac{1+\alpha}{2 \alpha}\right)^{2} \frac{\partial^{2} w}{\partial \theta^{2}} & =\frac{1+\alpha}{2 \alpha} u^{\frac{1-\alpha}{2 \alpha}} \frac{\partial^{2} u}{\partial \theta^{2}}+\frac{1-\alpha^{2}}{4 \alpha^{2}} u^{\frac{1-3 \alpha}{2 \alpha}}\left(\frac{\partial u}{\partial \theta}\right)^{2} \\
& =-\frac{1+\alpha}{2 \alpha} w+\frac{1+\alpha}{2 \alpha} w^{-1}+\frac{1-\alpha^{2}}{4 \alpha^{2}}\left(w^{\frac{1-3 \alpha}{1+\alpha}} E-w+\frac{2 \alpha}{\alpha-1} w^{-1}\right) \\
& =-\left(\frac{1+\alpha}{2 \alpha}\right)^{2} w+\frac{1-\alpha^{2}}{4 \alpha^{2}} E w^{\frac{1-3 \alpha}{1+\alpha}}
\end{aligned}
$$

where $E$ is given by (2.6) $)$, so that

$$
\frac{\partial^{2} w}{\partial \theta^{2}}+w=\frac{1-\alpha}{1+\alpha} E w^{\frac{1-3 \alpha}{1+\alpha}} .
$$

Scaling $w$ by a constant factor gives a solution of (2.3) with $\alpha$ replaced by $\frac{\alpha+1}{3 \alpha-1}$, and satisfying $w\left(\frac{1+\alpha}{2 \alpha} \Theta\right)=r^{\frac{1+\alpha}{2 \alpha}} w(0), w^{\prime}(0)=w^{\prime}\left(\frac{1+\alpha}{2 \alpha} \Theta\right)=0$. Therefore $\frac{1+\alpha}{2 \alpha} \Theta=$ $\Theta\left(\frac{\alpha+1}{3 \alpha-1}, r^{\frac{1+\alpha}{2 \alpha}}\right)$.

Similar results hold for the other period functions defined in Section 2, with essentially the same proof: 
Theorem 3.2. $\Xi$ is continuous on $(-\infty,-1) \times(0, \infty) \cup(1, \infty) \times(0, \infty)$, and $\Psi$ is continuous on $(0,1] \times(0, \infty)$, and the following limits hold:

$$
\begin{array}{ll}
\lim _{\alpha \searrow 1} \Xi(\alpha, v)=\frac{\pi}{2}, & v>0 ; \\
\lim _{\alpha \nearrow-1} \Xi(\alpha, v)=\frac{\pi}{2 v}, & v>0 ; \\
\lim _{\alpha \rightarrow \pm \infty} \Xi(\alpha, v)=\pi-2 \arctan v, & v>0 ; \\
\Xi(\alpha, 1)=\frac{\pi}{2}, & \alpha \in(-\infty,-1) \cup(1, \infty) ; \\
\lim _{v \searrow 0} \Xi(\alpha, v)=\frac{\pi \alpha}{1+\alpha}, & \alpha \in(-\infty,-1) \cup(1, \infty) ; \\
\lim _{v \nearrow \infty} \Xi(\alpha, v)=0, & \alpha \in(-\infty,-1) \cup(1, \infty) ; \\
\lim _{\lambda \searrow 0} \Psi(\alpha, \lambda)=\frac{\pi}{2}, & \alpha \in(0,1) ; \\
\lim _{\lambda \nearrow \infty} \Psi(\alpha, \lambda)=0, & \alpha \in(0,1) ; \\
\lim _{\alpha \searrow 0} \Psi(\alpha, \lambda)=0, & \lambda>0 .
\end{array}
$$

Furthermore the following symmetry holds:

$$
\Xi(\alpha, v)=\frac{2 \alpha}{1+\alpha} \Psi\left(\frac{\alpha+1}{3 \alpha-1}, \frac{\alpha-1}{\alpha+1} v^{2}\right)
$$

for $|\alpha|>1$ and $v>0$.

The symmetry (3.1) allows values of $\Theta$ for $\alpha<-1$ to be deduced from those with $\alpha \in(0,1 / 3)$, and those for $\alpha>1$ from those with $\alpha \in(1 / 3,1)$. Underlying this is the surprising fact that the support function of a homothetic solution for some $\alpha$, when transformed by taking a suitable power and scaling the argument, gives the support function of a homothetic solution for another value of $\alpha$. The symmetry (3.2) reflects the fact that the support function of a degenerate homothetically contracting solution for $\alpha>1$, taken to an appropriate power and with a suitable rescaling of its argument, gives the support function of a homothetically expanding solution for some $\alpha \in(1 / 3,1)$. Similarly, singular homothetically expanding solutions for $\alpha<-1$ can be obtained from homothetically expanding solutions for $\alpha \in(0,1 / 3)$.

\section{Monotonicity in $\alpha$}

In this section it is proved that $\Theta$ is monotone increasing in $\alpha$. This is immediate for the functions $\Xi$ and $\Psi$, since the corresponding integrands are clearly monotone increasing in $\alpha$ for fixed $v$.

To show that $\Theta(\alpha, r)$ is monotone in $\alpha$, denote

$$
I(\alpha, r, x)=\left(\frac{r^{2}-r^{\frac{\alpha-1}{\alpha}}}{1-r^{\frac{\alpha-1}{\alpha}}}-x^{2}-\frac{r^{2}-1}{1-r^{\frac{\alpha-1}{\alpha}}} x^{\frac{\alpha-1}{\alpha}}\right),
$$

and observe that if either $\alpha>\bar{\alpha}>1$ or $1>\alpha>\bar{\alpha}>0$ or $-1>\alpha>\bar{\alpha}$ then

$$
I(\alpha, r, x)-I(\bar{\alpha}, r, x)=\frac{r^{2}-r^{\frac{\alpha-1}{\alpha}}}{1-r^{\frac{\alpha-1}{\alpha}}}-\frac{r^{2}-r^{\frac{\bar{\alpha}-1}{\bar{\alpha}}}}{1-r^{\frac{\bar{\alpha}-1}{\alpha}}}-\frac{r^{2}-1}{1-r^{\frac{\alpha-1}{\alpha}}} x^{\frac{\alpha-1}{\alpha}}+\frac{r^{2}-1}{1-r^{\frac{\bar{\alpha}-1}{\alpha}}} x^{\frac{\bar{\alpha}-1}{\bar{\alpha}}} .
$$


This is zero for $x=1$ and for $x=r$. A direct computation gives

$$
x^{\frac{1}{\bar{\alpha}-\frac{1}{\alpha}+1}} \frac{\partial}{\partial x} x^{\frac{1}{\alpha}} \frac{\partial}{\partial x}(I(\alpha, r, x)-I(\bar{\alpha}, r, x))=\frac{\bar{\alpha}-1}{\bar{\alpha}} \frac{r^{2}-1}{r^{\frac{\bar{\alpha}-1}{\alpha}}-1} \frac{\bar{\alpha}-\alpha}{\alpha \bar{\alpha}}>0,
$$

from which it follows that $I(\alpha, r, x)-I(\bar{\alpha}, r, x)$ can have only minima as critical points in $x$, and therefore that $I(\alpha, r, x)<I(\bar{\alpha}, r, x)$ for all $x \in(1, r)$. It follows that

$$
\Theta(\alpha, r)-\Theta(\bar{\alpha}, r)=\int_{1}^{r}\left(\frac{1}{\sqrt{I(\alpha, r, x)}}-\frac{1}{\sqrt{I(\bar{\alpha}, r, x)}}\right) d x>0 .
$$

The monotonicity statement follows. This implies that there are no non-circular embedded homothetically contracting solutions for $\alpha>1 / 3$ : First, $\Theta(1 / 3, r)=\pi / 2$ for every $r$. Therefore by monotonicity, $\Theta(\alpha, r)>\pi / 2$ for every $r$. By the fourvertex theorem, an embedded closed curve must have at least four vertices, and so (if non-circular) must be constructed from at least four copies of the elementary curve segments. But then the total curvature is at least $4 \Theta(\alpha, r)$, but this is impossible since the total curvature must be $2 \pi$. This proves the first statement of Theorem 1.5, and Corollary 1.6

\section{Monotonicity in $r$}

The classification of embedded homothetic solutions for $\alpha<\frac{1}{3}$ requires a more difficult monotonicity result: The function $\Theta(\alpha, r)$ is monotone increasing in $r$ for $0<\alpha<\frac{1}{3}$. The proof also yields the fact that $\Theta(\alpha, r)$ is monotone decreasing in $r$ for $\frac{1}{3}<\alpha \leq 1$, and this implies by the symmetry (3.1) that $\Theta(\alpha, r)$ is monotone decreasing in $r$ for $\alpha>1$ and monotone increasing in $r$ for $\alpha<-1$.

A complication in this case compared to the argument in Section 4 is that the integrals defining $\Theta(\alpha, r)$ for different values of $r$ are taken over different domains. The first step is to transform to the same domain. There are many ways to do this, including the following natural family of transformations from $(1, r)$ to $(-1,1)$ : Let $\beta \in \mathbb{R} \backslash\{0\}$, and set $x^{\beta}=v$, where

$$
v=\frac{r^{\beta}-1}{2} z+\frac{r^{\beta}+1}{2} .
$$

Then the change of variables formula implies that

$$
\Theta(\alpha, r)=\int_{-1}^{1} \frac{d z}{\sqrt{\tilde{I}(\alpha, \beta, r, z)}},
$$

where the integrand is given for $\alpha \neq 1$ by

$$
\tilde{I}(\alpha, \beta, r, z)=\frac{4 \beta^{2}}{\left(r^{\beta}-1\right)^{2}}\left(\frac{r^{2}-r^{\frac{\alpha-1}{\alpha}}}{1-r^{\frac{\alpha-1}{\alpha}}} v^{\frac{2(\beta-1)}{\beta}}-v^{2}-\frac{r^{2}-1}{1-r^{\frac{\alpha-1}{\alpha}}} v^{2-\frac{\alpha+1}{\alpha \beta}}\right),
$$

and if $\alpha=1$ then

$$
\tilde{I}(1, \beta, r, z)=\frac{4 \beta^{2}}{\left(r^{\beta}-1\right)^{2}}\left(v^{\frac{2(\beta-1)}{\beta}}-v^{2}+\frac{r^{2}-1}{\beta \log r} v^{\frac{2(\beta-1)}{\beta}} \log v\right) .
$$

The aim is to show that $\tilde{I}(\alpha, \beta, r, z)$ is monotone in $r$ for each $z$. In order to choose $\beta$, consider the behaviour of $\bar{I}$ when $r$ is close to 1 :

$$
\tilde{I}(\alpha, \beta, r, z)=\left(\frac{1+\alpha}{\alpha}\right)\left(1-z^{2}\right)\left(1+\frac{z}{2}\left(\beta-\left(\frac{1+3 \alpha}{3 \alpha}\right)\right)(r-1)\right)+O\left((r-1)^{2}\right) \text {. }
$$


Thus the only possibility allowing monotonicity in $r$ for every $z$ is $\beta=\frac{1+3 \alpha}{3 \alpha}$.

Theorem 5.1. Define $J(\alpha, r, z)=\tilde{I}\left(\alpha, \frac{1+3 \alpha}{3 \alpha}, r, z\right)$. If $\alpha \in\left(0, \frac{1}{3}\right)$, then $\frac{\partial}{\partial r} J(\alpha, r, z)$ $<0$ for each $r>1$ and $z \in(-1,1)$. If $\alpha \in\left(\frac{1}{3}, 1\right]$ then $\frac{\partial}{\partial r} J(\alpha, r, z)>0$ for each $r>1$ and $z \in(-1,1)$.

As a first step in the proof, observe that $J(\alpha, r, z)$ is zero when $z= \pm 1$ for any $r$, so $\frac{\partial J}{\partial r}$ is zero when $z= \pm 1$. Therefore it suffices to show that $\frac{\partial J}{\partial r}$ is concave in $z$ for $\alpha \in\left(\frac{1}{3}, 1\right]$ and convex for $0<\alpha<\frac{1}{3}$, for each $r>1$ and $|z|<1$.

$\frac{\partial^{2} J}{\partial z^{2}}$ has the following surprisingly simple expression:

$$
\frac{1}{\beta^{2}} \frac{\partial^{2} J}{\partial z^{2}}= \begin{cases}-2+\frac{2(1-3 \alpha)}{(1+3 \alpha)^{2}}\left(\frac{r^{2}-r^{1-1 / \alpha}}{1-r^{1-1 / \alpha}} v^{-\frac{6 \alpha}{1+3 \alpha}}-\frac{r^{2}-1}{1-r^{1-1 / \alpha}} v^{-\frac{3(1+\alpha)}{1+3 \alpha}}\right), & \alpha \neq 1 \\ -2-\frac{1}{4}\left(v^{-3 / 2}+\frac{3\left(r^{2}-1\right)}{4 \log r} v^{-3 / 2} \log v\right), & \alpha=1\end{cases}
$$

The required convexity and concavity properties for $\frac{\partial J}{\partial r}$ therefore amount to monotonicity in $r$ of the quantity $Q$ defined by

$$
Q= \begin{cases}\frac{r^{2}-r^{\frac{\alpha-1}{\alpha}}}{1-r^{\frac{\alpha-1}{\alpha}}} v^{-\frac{6 \alpha}{1+3 \alpha}}-\frac{r^{2}-1}{1-r^{\frac{\alpha-1}{\alpha}}} v^{-\frac{3(1+\alpha)}{1+3 \alpha}}, & \alpha \neq 1, \\ v^{-\frac{3}{2}}+\frac{3\left(r^{2}-1\right)}{4 \log r} v^{-\frac{3}{2}} \log v, & \alpha=1 .\end{cases}
$$

Lemma 5.2. $Q=1$ when $z= \pm 1$.

Proof. When $z=-1, v=1$ and $Q=\frac{r^{2}-r^{1-1 / \alpha}-r^{2}+1}{1-r^{1-1 / \alpha}}=1$. When $z=1, v=r^{\frac{1+3 \alpha}{3 \alpha}}$, so $Q=\frac{r^{-2}\left(r^{2}-r^{1-1 / \alpha}\right)-r^{-(1+1 / \alpha)}\left(r^{2}-1\right)}{1-r^{1-1 / \alpha}}=1$.

Lemma 5.3. For $\alpha \in(0,1 / 3)$,

$$
\frac{\partial}{\partial z}\left(v^{\frac{2(3 \alpha-1)}{1+3 \alpha}} \frac{\partial}{\partial z}\left(v^{\frac{3(1+\alpha)}{1+3 \alpha}} \frac{\partial Q}{\partial r}\right)\right)<0
$$

whenever $|z|<1$ and $r>1$, while for $\alpha \in(1 / 3,1]$

$$
\frac{\partial}{\partial z}\left(v^{2} \frac{\partial}{\partial z}\left(v^{\frac{3(1+\alpha)}{1+3 \alpha}} \frac{\partial Q}{\partial r}\right)\right)<0
$$

whenever $|z|<1$ and $r>1$.

Proof. First assume that $\alpha \neq 1$. Equation (5.7) yields the following expression for $\frac{\partial Q}{\partial r}$, since $\frac{\partial v}{\partial r}=\frac{1+3 \alpha}{3 \alpha} \frac{r^{\frac{1}{3 \alpha}}}{r^{\frac{1+3 \alpha}{3 \alpha}}-1}(v-1)$ :

$$
\begin{aligned}
\frac{\partial Q}{\partial r}= & \left\{\left(r^{\frac{1+3 \alpha}{3 \alpha}}-1\right)^{\frac{6 \alpha}{1+3 \alpha}} \frac{\partial}{\partial r}\left(\frac{r^{2}-r^{\frac{\alpha-1}{\alpha}}}{\left(1-r^{\frac{\alpha-1}{\alpha}}\right)\left(r^{\frac{1+3 \alpha}{3 \alpha}}-1\right)^{\frac{6 \alpha}{1+3 \alpha}}}\right)\right\} v^{-\frac{6 \alpha}{1+3 \alpha}} \\
& -\left\{\left(r^{\frac{1+3 \alpha}{3 \alpha}}-1\right)^{\frac{3(1+\alpha)}{1+3 \alpha}} \frac{\partial}{\partial r}\left(\frac{r^{2}-1}{\left(1-r^{\frac{\alpha-1}{\alpha}}\right)\left(r^{\frac{1+3 \alpha}{3 \alpha}}-1\right)^{\frac{3(1+\alpha)}{1+3 \alpha}}}\right)\right\} v^{-\frac{3(1+\alpha)}{1+3 \alpha}} \\
& +\frac{r^{\frac{1}{3 \alpha}}}{r^{\frac{1+3 \alpha}{3 \alpha}}-1}\left(2 \frac{r^{2}-r^{\frac{\alpha-1}{\alpha}}}{1-r^{\frac{\alpha-1}{\alpha}}} v^{-\frac{1+9 \alpha}{1+3 \alpha}}-\frac{1+\alpha}{\alpha} \frac{r^{2}-1}{1-r^{\frac{\alpha-1}{\alpha}}} v^{-\frac{4+6 \alpha}{1+3 \alpha}}\right) .
\end{aligned}
$$


Multiplication by $v^{\frac{3(1+\alpha)}{1+3 \alpha}}$ and differentiation with respect to $z$ yields

$$
\begin{aligned}
\frac{2}{r^{\frac{1+3 \alpha}{3 \alpha}}-1} & \frac{\partial}{\partial z}\left(v^{\frac{3(1+\alpha)}{1+3 \alpha}} \frac{\partial Q}{\partial r}\right) \\
= & \frac{3(1-\alpha)}{1+3 \alpha}\left\{\left(r^{\frac{1+3 \alpha}{3 \alpha}}-1\right)^{\frac{6 \alpha}{1+3 \alpha}} \frac{\partial}{\partial r}\left(\frac{r^{2}-r^{\frac{\alpha-1}{\alpha}}}{\left(1-r^{\frac{\alpha-1}{\alpha}}\right)\left(r^{\frac{1+3 \alpha}{3 \alpha}}-1\right)^{\frac{6 \alpha}{1+3 \alpha}}}\right)\right\} v^{\frac{2(1-3 \alpha)}{1+3 \alpha}} \\
& +\frac{4(1-3 \alpha)}{(1+3 \alpha)} \frac{r^{\frac{1}{3 \alpha}}}{r^{\frac{1+3 \alpha}{3 \alpha}}-1}\left(\frac{r^{2}-r^{\frac{\alpha-1}{\alpha}}}{1-r^{\frac{\alpha-1}{\alpha}}}\right) v^{\frac{1-9 \alpha}{1+3 \alpha}} \\
& +\frac{1+\alpha}{\alpha} \frac{r^{\frac{1}{3 \alpha}}}{r^{\frac{1+3 \alpha}{3 \alpha}}-1}\left(\frac{r^{2}-1}{1-r^{\frac{\alpha-1}{\alpha}}}\right) v^{-2}
\end{aligned}
$$

Assume $0<\alpha<\frac{1}{3}$. Multiplication by $v^{\frac{2(3 \alpha-1)}{1+3 \alpha}}$ and differentiation with respect to $z$ kills the first term and yields

$$
\begin{aligned}
\frac{4 r^{-\frac{1}{3 \alpha}}}{r^{\frac{1+3 \alpha}{3 \alpha}}-1} \frac{\partial}{\partial z}\left(v^{\frac{2(3 \alpha-1)}{1+3 \alpha}} \frac{\partial}{\partial z}\left(v^{\frac{3(1+\alpha)}{1+3 \alpha}} \frac{\partial Q}{\partial r}\right)\right)= & -\frac{4(1-3 \alpha)}{1+3 \alpha}\left(\frac{r^{2}-r^{\frac{\alpha-1}{\alpha}}}{1-r^{\frac{\alpha-1}{\alpha}}}\right) v^{-2} \\
& -\frac{4(1+\alpha)}{\alpha(1+3 \alpha)}\left(\frac{r^{2}-1}{1-r^{1-\frac{1}{\alpha}}}\right) v^{-\frac{5+3 \alpha}{1+3 \alpha}}
\end{aligned}
$$

The right-hand side of (5.10) is manifestly negative for $r>1$ and $|z|<1$ (i.e. $1<v<r)$, so the first statement of Lemma 5.3 is proved.

Now consider the case $\alpha \in\left(\frac{1}{3}, 1\right)$ : Multiplication of Equation (5.9) by $v^{2}$ and differentiation with respect to $z$ yields:

$$
\begin{aligned}
\frac{(1+3 \alpha)^{2}}{r^{\frac{1+3 \alpha}{3 \alpha}}-1} & \frac{\partial}{\partial z}\left(v^{2} \frac{\partial}{\partial z}\left(v^{\frac{3(1+\alpha)}{1+3 \alpha}} \frac{\partial Q}{\partial r}\right)\right) \\
= & 3(1+\alpha)(1-3 \alpha) r^{\frac{1}{3 \alpha}}\left(\frac{r^{2}-r^{-\frac{1-\alpha}{\alpha}}}{1-r^{-\frac{1-\alpha}{\alpha}}}\right) v^{\frac{3(1-\alpha)}{1+3 \alpha}} \\
& +3(1-\alpha)\left(r^{\frac{1+3 \alpha}{3 \alpha}}-1\right)^{\frac{1+9 \alpha}{1+3 \alpha}} \frac{\partial}{\partial r}\left(\frac{1-r^{-\frac{1+\alpha}{\alpha}}}{\left(1-r^{-\frac{1-\alpha}{\alpha}}\right)\left(1-r^{-\frac{1+3 \alpha}{3 \alpha}}\right)^{\frac{6 \alpha}{1+3 \alpha}}}\right) v^{\frac{2}{1+3 \alpha}} .
\end{aligned}
$$

The first term on the right-hand side of (5.11) is negative. The bracket in the second term is the product of the two positive decreasing functions

$$
\frac{1-r^{-\frac{1+\alpha}{\alpha}}}{1-r^{-\frac{1-\alpha}{\alpha}}} \quad \text { and } \quad\left(1-r^{-\frac{1+3 \alpha}{3 \alpha}}\right)^{-\frac{6 \alpha}{1+3 \alpha}}
$$

and so is positive and decreasing. Therefore the second term in Equation (5.11) is negative for $|z|<1$ and $r>1$ for $\alpha \in\left(\frac{1}{3}, 1\right)$. 
The case $\alpha=1$ is similar to that for $\alpha \in\left(\frac{1}{3}, 1\right)$ : The analogous computations in this case lead to the following:

$$
\begin{aligned}
\frac{\partial}{\partial z}\left(v^{2} \frac{\partial}{\partial z}\left(v^{\frac{3(1+\alpha)}{1+3 \alpha}} \frac{\partial Q}{\partial r}\right)\right)= & -\frac{3}{8} r^{\frac{1}{3}}\left(r^{\frac{4}{3}}-1\right) \frac{r^{2}-1}{\log r} v^{-1} \\
& +\frac{3}{16}\left(r^{\frac{4}{3}}-1\right)^{\frac{7}{2}} \frac{\partial}{\partial r}\left(\frac{1-r^{-2}}{\log r\left(1-r^{-\frac{4}{3}}\right)^{\frac{3}{2}}}\right) .
\end{aligned}
$$

As in the previous case, the first term is manifestly negative, and the bracket in the second is the product of the two positive decreasing functions $\frac{1-r^{-2}}{\log r}$ and $\left(1-r^{-\frac{4}{3}}\right)^{-\frac{3}{2}}$, hence decreasing, so the left-hand side is negative whenever $r>1$ and $|z|<1$.

Lemma 5.4. $\frac{\partial Q}{\partial r}>0$ for any $\alpha \in(0,1],|z|<1$ and $r>1$.

Proof. Consider first the case $0<\alpha<\frac{1}{3}$. By Lemma 5.2, $\frac{\partial Q}{\partial r}=0$ whenever $z= \pm 1$, and hence also $v^{\frac{3(1+\alpha)}{1+3 \alpha}} \frac{\partial Q}{\partial r}=0$ when $z= \pm 1$. If there exists $r>1$ and $z \in(-1,1)$ such that $\frac{\partial Q}{\partial r}<0$, then there exists $z$ such that $v^{\frac{3(1+\alpha)}{1+3 \alpha}} \frac{\partial Q}{\partial r}$ has a negative minimum (for the same value of $r$ ). But then at this point

$$
\frac{\partial}{\partial z}\left(v^{\frac{3(1+\alpha)}{1+3 \alpha}} \frac{\partial Q}{\partial r}\right)=0
$$

and

$$
\frac{\partial^{2}}{\partial z^{2}}\left(v^{\frac{3(1+\alpha)}{1+3 \alpha}} \frac{\partial Q}{\partial r}\right) \geq 0
$$

so that

$$
\frac{\partial}{\partial z}\left(v^{\frac{2(3 \alpha-1)}{1+3 \alpha}} \frac{\partial}{\partial z}\left(v^{\frac{3(1+\alpha)}{1+3 \alpha}} \frac{\partial Q}{\partial r}\right)\right) \geq 0,
$$

in contradiction to Lemma 5.3. The cases $\frac{1}{3}<\alpha \leq 1$ are similar.

Lemma 5.5. If $|z|<1$ and $r>1$ and $\alpha \in\left(0, \frac{1}{3}\right)$, then $\frac{\partial^{3} J}{\partial r \partial z^{2}}>0$. If $|z|<1$ and $r>1$ and $\alpha \in\left(\frac{1}{3}, 1\right]$, then $\frac{\partial^{3} J}{\partial r \partial z^{2}}<0$.

Proof. This is immediate by Equation (5.6) and Lemma 5.4.

Since $\frac{\partial J}{\partial r}=0$ whenever $z= \pm 1$, Lemma 5.5 implies the theorem.

Corollary 5.6. $\Theta(\alpha, r)$ is strictly decreasing in $r$ for $\alpha>\frac{1}{3}$, and strictly increasing in $r$ for $\alpha \in\left(0, \frac{1}{3}\right)$ or $\alpha<-1$, for every $r>1$.

Proof. It follows from Theorem 5.1 and Equation (5.2) that $\Theta$ is strictly monotone decreasing in $r$ for $\alpha \in\left(\frac{1}{3}, 1\right]$, and strictly monotone increasing in $r$ for $\alpha \in\left(0, \frac{1}{3}\right)$, for any $r>1$. The remaining cases follow from the symmetry (3.1).

Corresponding results for the functions $\Xi$ and $\Psi$ also hold, and are rather easy to prove (see [23]). 


\section{Proofs of Theorems 1.5 and 1.7}

The main theorems can be deduced from the monotonicity statement of Corollary 5.6. First the embedded case: An embedded homothetic solution has total curvature $2 \pi$, so the period $\Theta(\alpha, r)$ must equal $\frac{\pi}{k}$ for some integer $k$. For fixed $\alpha$, the values of $\Theta(\alpha, r)$ lie between $\pi \sqrt{\frac{\alpha}{1+\alpha}}$ and $\frac{\pi}{2}$. This never equals $\pi$, and equals $\frac{\pi}{2}$ only for $\alpha=\frac{1}{3}$. If $k \geq 3$ it equals $\frac{\pi}{k}$ for some $r>1$ if and only if $0<\alpha<\frac{1}{k^{2}-1}$. Furthermore the monotonicity result implies that for $\alpha$ in this range there is precisely one value of $r$ for which $\Theta(\alpha, r)$ has the correct value.

The proof of Theorem 1.7 is similar: In order to have a homothetic solution with total curvature $2 n \pi$ and $m$ maxima of curvature, the total curvature of the portion between consecutive critical points must be $\frac{\pi n}{m}$, and this must equal $\Theta(\alpha, r)$ for some $r>1$. The argument proceeds as above.

\section{Applications}

The results of the paper have some implications for the behaviour of the flows when $0<\alpha<\frac{1}{3}$. The first is an improvement of the main result of [6] for the isotropic case. Denote by $\mathcal{K}^{(j)}$ the space of $C^{j}$ convex regions in the plane, with the topology of $C^{j}$ convergence of support functions.

Theorem 7.1. Let $\alpha \in\left(0, \frac{1}{3}\right)$. Then for any $j \geq 0$ there exists an open dense subset $\mathcal{S}$ in $\mathcal{K}^{(j)}$ such that solutions with initial data in $\mathcal{S}$ have isoperimetric ratio approaching infinity as $t \rightarrow T$.

This is an improvement of the result in [6] in two senses: First, the set $\mathcal{S}$ is open and dense rather than generic. Second, there is no requirement of symmetry on the solutions. The proof is identical to that in [6] except for two observations: The first improvement follows immediately from the fact that there are only finitely many homothetic solutions (up to rotation) for each $\alpha$. The second improvement comes from the observation that for $0<\alpha<\frac{1}{3}$ all homothetic solutions are unstable, not only the symmetric ones as was proved in 6 .

The key ingredient in the proof from [6] is the linear instability of homothetic solutions. This is determined by a stability eigenvalue $\lambda$, which has the following variational characterisation (modified from [6] to factor out the direction of neutral stability corresponding to rotations, as well as the directions corresponding to dilations and translations):

$$
\lambda=\inf _{C^{\infty}\left(S^{1}\right)}\left\{\frac{\int_{S^{1}} f_{\theta}^{2}-f^{2}}{\int_{S^{1}} u^{-\frac{1+\alpha}{\alpha}} f^{2}} \mid \int u^{-\frac{1}{\alpha}} f=\int u^{-\frac{1+\alpha}{\alpha}} u_{\theta} f=\int u^{-\frac{1+\alpha}{\alpha}} f \cos \left(\theta-\theta_{0}\right)=0\right\} .
$$

A homothetic solution is linearly unstable if $\lambda<\frac{1}{\alpha}$. $\lambda$ is continuous and is equal to 3 on $S^{1}$, hence, it is less than $\frac{1}{\alpha}$ for $0<\alpha<\frac{1}{3}$. It suffices to show that $\lambda$ is never equal to $\frac{1}{\alpha}$ along the curves of solutions $\Gamma_{k, \alpha}$ as $\alpha$ decreases below $1 /\left(k^{2}-1\right)$. If $\lambda$ equals $\frac{1}{\alpha}$ at some point, then there exists a function $f$ (the minimizer of the Rayleigh quotient) which satisfies

$$
\eta_{\theta \theta}+\eta+\frac{1}{\alpha} u^{-\frac{1+\alpha}{\alpha}} \eta=0
$$

on $S^{1}$, linearly independent of the function $u_{\theta}$. 
Lemma 7.2. Let $k \geq 3$ and $0<\alpha<1 /\left(k^{2}-1\right)$. If $u$ is the support function of $\Gamma_{k, \alpha}$, so that

$$
\begin{aligned}
u_{\theta \theta}+u & =u^{-\frac{1}{\alpha}}, \\
u_{\theta}(0) & =0, \\
u_{\theta}(\pi / k) & =0, \\
u_{\theta}(\theta) & <0, \quad 0<\theta<\pi / k,
\end{aligned}
$$

and $\eta$ satisfies

$$
\begin{aligned}
\eta_{\theta \theta}+\eta+\frac{1}{\alpha} u^{-\frac{1+\alpha}{\alpha}} \eta & =0, \\
\eta(0) & >0 \\
\eta_{\theta}(0) & =0
\end{aligned}
$$

then $\eta_{\theta}(2 \pi)>0$.

Proof. Consider the function $U(\alpha, r, \theta)$ defined by

$$
\begin{aligned}
U_{\theta \theta}+U & =U^{-\frac{1}{\alpha}}, \\
U_{\theta}(\alpha, r, 0) & =0, \\
U_{\theta}(\alpha, r, \Theta(\alpha, r)) & =0, \\
U_{\theta}(\alpha, r, \theta) & <0, \quad 0<\theta<\Theta(\alpha, r), \\
U(\alpha, r, 0) & =r U(\alpha, r, \Theta(\alpha, r)) .
\end{aligned}
$$

Let $\eta(\theta)=\frac{d}{d r} U(\alpha, r, \theta)$, so (7.2) holds with $\eta(0)>0$ and $\eta_{\theta}(0)=0$. The derivative with respect to $r$ of the identity $U_{\theta}(\alpha, r, 2 k \Theta(\alpha, r))=0$ gives

$$
\eta_{\theta}(2 k \Theta(\alpha, r))+2 k U_{\theta \theta}(\alpha, r, 2 k \Theta(\alpha, r)) \frac{d}{d r} \Theta(\alpha, r)=0 .
$$

By Corollary 16, $\frac{d}{d r} \Theta(\alpha, r)>0$. Also, $U(\alpha, r,$.$) has a local maximum at \theta=$ $2 k \Theta(\alpha, r)$, so $U_{\theta \theta}<0$. Therefore $\eta_{\theta}(2 \pi)=\eta_{\theta}(2 k \Theta(\alpha, r))>0$.

Lemma 7.3. If $u$ is the support function of $\Gamma_{k, \alpha}$, then the only functions $\eta$ on $S^{1}$ satisfying Equation (7.2) have the form $\eta=c u_{\theta}$.

Proof. The solutions of Equation (7.2) are $f=A u_{\theta}+B \eta$, where $\eta$ is defined in Lemma 7.2. But then $f_{\theta}(2 \pi)=A u_{\theta \theta}(2 \pi)+B \eta_{\theta}(2 \pi) \neq A u_{\theta \theta}(0)=f_{\theta}(0)$ if $B \neq 0$, by the result of Lemma 7.2. Therefore the only solution with periodic boundary conditions on $[0,2 \pi]$ is that with $B=0$.

Lemma 7.4. All homothetic solutions are linearly unstable for $0<\alpha<\frac{1}{3}$.

Proof. For the circle solution $\lambda=3<\frac{1}{\alpha}$ and, by continuity, $\lambda<\frac{1}{\alpha}$ also for $\Gamma_{k, \alpha}$ with $\alpha$ sufficiently close to $1 /\left(k^{2}-1\right)$. Lemma 7.3 implies that $\lambda \neq \frac{1}{\alpha}$ along $\left\{\Gamma_{k, \alpha}: 0<\alpha<1 /\left(k^{2}-1\right)\right\}$. It follows that $\lambda<\frac{1}{\alpha}$ along this curve, and therefore $\Gamma_{k, \alpha}$ is always linearly unstable.

The proof from [6] applies to give Theorem [7.1

The next application is to the behaviour of $k$-fold symmetric solutions. In this case some immersed cases can also be handled. Denote

$$
\mathcal{K}_{n, k}^{(\ell)}=\left\{u \in C^{\ell}(\mathbb{R}): u\left(\theta+2 \pi \frac{n}{k}\right)=u(\theta), u_{\theta \theta}+u>0\right\} .
$$


$\mathcal{K}_{n, k}^{(\ell)}$ therefore consists of the support functions of $C^{\ell}$ locally convex curves with total curvature $2 n \pi$ and a $k$-fold rotation symmetry.

Theorem 7.5. Let $n$ and $k$ be integers such that $k>2 n$. If $\alpha<0$ or $\alpha \geq \frac{n^{2}}{k^{2}-n^{2}}$ then all solutions with initial data in $\mathcal{K}_{n, k}^{(\ell)}$ converge to circles after rescaling. If $0<\alpha<\frac{n^{2}}{k^{2}-n^{2}}$ then there is an open dense set $\mathcal{S}$ in $\mathcal{K}_{n, k}^{(\ell)}$ such that solutions with initial data in $\mathcal{S}$ converge after rescaling to the homothetic solution $\Gamma_{n, k, \alpha}$ defined in Theorem 1.7

The case of embedded $k$-fold symmetric solutions has $n=1, k \geq 3$.

Proof. The proof has several steps: First, uniform bounds on the curvature and higher derivatives of the rescaled solutions are established. Then it is shown that the rescaled solutions approach a homothetic solution as the final time is approached. The results for $\alpha<0$ and $\alpha \geq n^{2} /\left(k^{2}-n^{2}\right)$ follow since the circle is the only homothetic solution in $\mathcal{K}_{n, k}^{(\ell)}$ for this range of $\alpha$, by Theorem 1.7. The results for smaller $\alpha$ will follow from an argument similar to that given for Theorem 7.1 ,

The key to the regularity estimates is a simple isoperimetric bound which is a consequence of the $k$-fold rotation symmetry. It is here that the condition $k>2 n$ is crucial:

Lemma 7.6. There exists a constant $C$ depending only on $n$ and $k$ such that every $u \in \mathcal{K}_{n, k}^{(\ell)}$ satisfies $\sup u \leq C \inf u$.

Proof. Let $u \in \mathcal{K}_{n, k}^{(\ell)}$, and rotate so that $u(0)=\sup u$. Then $u_{\theta}(0)=0$, and $u(2 \pi n / n)=u(0), u_{\theta}(2 \pi n / k)=0$. Let

$$
f(\theta)=u(\theta) \cos (\theta-\pi n / k)-u_{\theta}(\theta) \sin (\theta-\pi n / k) .
$$

Then $f(0)=f(2 \pi n / k)=u(0) \cos (\pi n / k)$. Compute the derivative:

$$
f_{\theta}(\theta)=-\left(u_{\theta \theta}+u\right) \sin (\theta-\pi n / k) .
$$

Since $u_{\theta \theta}+u>0$, it follows that $f$ is increasing on $[0, \pi n / k)$ and decreasing on $(\pi n / k, 2 \pi n / k]$. This implies that $f(\theta) \geq f(0)$ for $\theta \in[0,2 \pi n / k]$. At a point in $(0,2 \pi n / k)$ where $u$ attains its minimum, $u_{\theta}=0$, and therefore

$$
u(\theta) \cos (\theta-\pi n / k)=f(\theta) \geq u(0) \cos (\pi n / k) .
$$

This implies that inf $u=u(\theta) \geq u(0) \cos (\pi n / k)=\sup u \cos (\pi n / k)$. Note that since $k>2 n, 0<\pi n / k<\pi / 2$ and $\cos (\pi n / k)>0$.

Bounds on the speed now follow from results in [4]: For $\alpha<0$, bounds on the radius of curvature is provided by [4, Lemma I1.7 and Lemma I1.8], while for $\alpha>0$ bounds on curvature are obtained by following the proof of [4, Proposition II1.6]. Estimates on the support function in $C^{\infty}$ for $-1 \leq \alpha<0$, or $C^{2+1 /|\alpha|}$ for $\alpha<-1$, are provided by [4, Lemma I1.11], and estimates in $C^{\infty}$ for $0<\alpha<1$ or in $C^{2+\frac{1}{\alpha-1}}$ for $\alpha>1$ are given by [4, Theorem II1.9].

The convergence of rescaled solutions to homothetic solutions is a consequence of the following monotonicity result: 
Lemma 7.7. For any immersed compact solution of Equation (1.1),

$$
\frac{d}{d t}\left(\frac{\int u_{\theta}^{2}-u^{2} d \theta}{\left(\int u^{\frac{\alpha-1}{\alpha}} d \theta\right)^{\frac{2 \alpha}{\alpha-1}}}\right) \leq 0
$$

for $\alpha \neq 1$, while

$$
\frac{d}{d t}\left(\frac{\int u_{\theta}^{2}-u^{2} d \theta}{\exp \left(\frac{1}{n \pi} \int \log u d \theta\right)}\right) \leq 0
$$

for $\alpha=1$. Equality holds if and only if Equation (2.1) holds.

Proof. Under the evolution equation (1.1), the support function $u$ evolves according to the equation

$$
\frac{\partial u}{\partial t}=-\frac{1}{\alpha}\left(u_{\theta \theta}+u\right)^{-\alpha} .
$$

From this, the evolution equation for the integral quantities in the lemma can be computed:

$$
\begin{aligned}
\frac{d}{d t}\left(\int u_{\theta}^{2}-u^{2} d \theta\right) & =2 \int u_{\theta} u_{t \theta}-u u_{t} d \theta \\
& =-2 \int\left(u_{\theta \theta}+u\right) u_{t} d \theta \\
& =\frac{2}{\alpha} \int\left(u_{\theta \theta}+u\right)^{1-\alpha} d \theta
\end{aligned}
$$

while

$$
\frac{d}{d t}\left(\int u^{\frac{\alpha-1}{\alpha}} d \theta\right)=-\frac{\alpha-1}{\alpha^{2}} \int u^{-\frac{1}{\alpha}}\left(u_{\theta \theta}+u\right)^{-\alpha} d \theta .
$$

These two equations give the following:

$$
\begin{aligned}
\frac{d}{d t}\left(\frac{\int u_{\theta}^{2}-u^{2} d \theta}{\left(\int u^{\frac{\alpha-1}{\alpha}} d \theta\right)^{\frac{2 \alpha}{\alpha-1}}}\right)=\frac{2 \alpha^{-1}}{\left(\int u^{\frac{\alpha-1}{\alpha}} d \theta\right)^{\frac{3 \alpha-1}{\alpha-1}}} & \left(\int u^{\frac{\alpha-1}{\alpha}} d \theta \int\left(u_{\theta \theta}+u\right)^{1-\alpha} d \theta\right. \\
& \left.+\int u_{\theta}^{2}-u^{2} d \theta \int u^{-\frac{1}{\alpha}}\left(u_{\theta \theta}+u\right)^{-\alpha} d \theta\right) .
\end{aligned}
$$

Let $f=\left(u_{\theta \theta}+u\right) u^{\frac{1}{\alpha}}$ and $d \mu=u^{\frac{\alpha-1}{\alpha}} d \theta$. Then the quantity in brackets can be written as

$$
\int d \mu \int f^{1-\alpha} d \mu-\int f d \mu \int f^{-\alpha} d \mu .
$$

By the Hölder inequality, this is non-negative if $\alpha<0$, and non-positive if $\alpha>0$, with equality holding if and only if $f$ is constant.

The regularity results together with Lemma[7.7 imply that there is a subsequence of times approaching the final time for which the rescaled solutions converge to a homothetic solution. In view of the bounds above and below on the support function from Lemma 7.6 homothetic solutions are smooth and strictly convex, and therefore the methods of [3] apply to show that the rescaled solutions converge smoothly to the homothetic solution as $t$ approaches the final time. 
In the cases $\alpha<0$ and $\alpha>n^{2} /\left(k^{2}-n^{2}\right)$ this completes the proof, since the $n$-fold circle is the only homothetic solution. The final ingredient needed to deal with the remaining values of $\alpha$ is the following:

Lemma 7.8. If $0<\alpha<n^{2} /\left(k^{2}-n^{2}\right)$ then all homothetic solutions in $\mathcal{K}_{n, k}^{(\ell)}$ other than scaled translates of $\Gamma_{n, k, \alpha}$ are linearly unstable in $\mathcal{K}_{n, k}^{(\ell)}$.

Proof. This is proved in exactly the same manner as Lemma 7.4 The linear stability is determined by the stability eigenvalue

$$
\lambda=\inf _{f \in C^{\infty}(\mathbb{R} /(2 n \pi / k) \mathbb{Z})}\left\{\frac{\int f_{\theta}^{2}-f^{2}}{\int u^{-\frac{1+\alpha}{\alpha}} f^{2}} \mid \int u^{-\frac{1}{\alpha}} f=\int u^{-\frac{1+\alpha}{\alpha}} u_{\theta} f=0\right\} .
$$

If $\alpha<n^{2} /\left(k^{2}-n^{2}\right)$ then the $n$-fold circle solution is linearly unstable. The remaining homothetic solutions are scaled translates of $\Gamma_{n, j k, \alpha}$ for $j \geq 2$, and these occur for $0<\alpha<n^{2} /\left(j^{2} k^{2}-n^{2}\right)<n^{2} /\left(k^{2}-n^{2}\right)$. By continuity, $\lambda<1 / \alpha$ on $\Gamma_{n, j k, \alpha}$ for $\alpha$ sufficiently close to $n^{2} /\left(j^{2} k^{2}-n^{2}\right)$, and the argument of Lemma 7.2 and Lemma 7.3 implies that $\lambda$ never equals $1 / \alpha$ on $\Gamma_{n, j k, \alpha}$. Therefore these are all linearly unstable.

The proof from [6] now adapts to this situation readily: The increasing entropy used there is replaced by the scaling-invariant integral from Lemma 7.7, and Lemma 7.8 ensures that small perturbations can be made to any solution to avoid converging to homothetic solutions other than $\Gamma_{n, k, \alpha}$.

\section{REFERENCES}

[1] U. Abresch and J. Langer, The normalised curve shortening flow and homothetic solutions, J. Differential Geom. 23 (1986), 175-196. MR 88d:53001

[2] B. Andrews, Contraction of convex hypersurfaces by their affine normal, J. Differential Geom. 43 (1996), 207-230. MR 97m:58045

[3] _ Monotone quantities and unique limits for evolving convex hypersurfaces, Internat. Math. Res. Notices 20 (1997), 1001-1031. MR 99a:58041

[4] _ Evolving convex curves, Calc. Var. Partial Differential Equations 7 (1998), no 3, 315-371. MR 99k:58038

[5] Motion of hypersurfaces by Gauss curvature, Pacific J. Math. 195 (2000), 1-34. MR 2001i:53108

[6] - Nonconvergence and instability in the asymptotic behaviour of curves evolving by curvature, Comm. Anal. Geom. 10 (2002), 409-449.

[7] S. Angenent, G. Sapiro and A. Tannenbaum, On the affine heat equation for non-convex curves, J. Amer. Math. Soc. 11 (1998), 601-634. MR 99d:58039

[8] B. Chow and H.-D. Tsai, Geometric expansion of convex plane curves, J. Differential Geom. 44 (1996), 312-330. MR 97m:58041

[9] K.-S. Chou and X.-P. Zhu, "The Curve Shortening Problem", Chapman and Hall/CRC (2000).

[10] A convexity theorem for a class of anisotropic flows of plane curves, Indiana Univ. Math. J. 48 (1999), 139-154. MR 2000m:53098

[11] M. Gage, An isoperimetric inequality with applications to curve shortening, Duke Math. J. 50 (1983), 1225-1229. MR 85d:52007

[12] _ Curve shortening makes convex curves circular, Invent. Math. 76 (1984), 357-364. MR 85i:52004

[13] C. Gerhardt, Flow of nonconvex hypersurfaces into spheres, J. Differential Geom. 32 (1990), 299-314. MR 91k:53016

[14] M. Grayson, The heat equation shrinks embedded plane curves to round points, J. Differential Geom. 26 (1987), 285-314. MR 89b:53005 
[15] M. Gage and R. Hamilton, The heat equation shrinking convex plane curves, J. Differential Geom. 23 (1986), 69-96. MR 87m:53003

[16] R. Hamilton, Isoperimetric estimates for the curve shrinking flow in the plane, Ann. of Math. Stud. 137 (1995), 201-222. MR 96k:58043

[17] G. Huisken, A distance comparison principle for evolving curves, Asian J. Math. 2 (1998), 127-133. MR 99m:58052

[18] J. Oaks, Singularities and self-intersections of curves evolving on surfaces, Indiana Univ. Math. J. 43 (1994), 959-981. MR 95k:58039

[19] G. Sapiro and A. Tannenbaum, On affine plane curve evolution, J. Funct. Anal. 119 (1994), 79-120. MR 94m:58049]

[20] D.-H. Tsai, Geometric expansion of star-shaped plane curves, Comm. Anal. Geom. 4 (1996), 459-480. MR 97 m:58042

[21] J. Urbas, An expansion of convex hypersurfaces, J. Differential Geom. 33 (1991), 91-125; Correction to, ibid. 35, 763-765. MR 93b:58142

[22] - On the expansion of star-shaped hypersurfaces by symmetric functions of their principal curvatures, Math. Z. 205 (1990), 355-372. MR 92c:53037

[23] Convex curves moving homothetically by negative powers of their curvature, Asian J. Math. 3 (1999), 635-656. MR 2001m:53119

Centre for Mathematics and its Applications, Australian National University, ACT 0200, Australia

E-mail address: andrews@maths.anu.edu.au 